\title{
Type Ia Supernovae: Non-standard Candles of the Universe
}

\author{
A. I. Bogomazov ${ }^{1}$, A. V. Tutukov ${ }^{2}$ \\ ${ }^{1}$ Sternberg Astronomical Institute, Moscow State University, \\ Universitetskii pr. 13, Moscow, 119991 Russia \\ ${ }^{2}$ Institute of Astronomy, Russian Academy of Sciences, \\ Pyatnitskaya ul. 48, Moscow 119017, Russia
}

\begin{abstract}
We analyze the influence of the evolution of light absorption by gray dust in the host galaxies of type Ia supernovae (SNe Ia) and the evolution of the mean combined mass of close-binary carbon-oxygen white dwarfs merging due to gravitational waves (SNe Ia precursors) on the interpretation of Hubble diagrams for SNe Ia. A significant increase in the mean SNe Ia energy due to the higher combined masses of merging dwarfs should be observable at redshifts $z>2$. The observed relation between the distance moduli and redshifts of SNe Ia can be interpreted not only as evidence for accelerated expansion of the Universe, but also as indicating time variations of the gray-dust absorption of light from these supernovae in various types of host galaxies, observational selection effects, and the decrease in mean combined masses of merging degenerate dwarfs.
\end{abstract}

Astronomy Reports, v. 55, pp. 497-504 (2011)

\section{INTRODUCTION}

This paper continues our study [1], where we demonstrated that the mean combined masses of binary carbon-oxygen white dwarfs (CO WDs) merging due to gravitationalwave radiation should evolve with time. Paper [1] presents the results of population syntheses performed with the "Scenario Machine" (a computer code for evolutionary studies of close binaries), as well as computations of the merger rate and mass distributions of merging white dwarfs (WDs). Such studies are important because observations of distant type Ia supernovae (SNe Ia) in the late 1990's led to the conclusion that the expansion of the Universe was accelerating ${ }^{1}$ [2, 3]. Thus, understanding the physics of SNe Ia determining the possible evolution of their brightness with time is needed to understand the fundamental properties of the Universe. In addition, in contrast to our first study, we also consider here the influence of the evolution of gray dust in the host galaxies of SNe Ia on changes in the absorption of light from these supernovae. Light absorption by gray dust is able to produce the same effect of fainter distant supernovae as the proposed accelerated expansion of the Universe.

\footnotetext{
${ }^{1}$ At $z \approx 1$ were found to be fainter than expected for standard supernovae of this type by, on average, about $0^{m} \cdot 2[2,3$.
} 
There are two main models for the origin of a SNe Ia outburst. The first requires a close binary consisting of a CO WD and a non-degenerate star, whose matter is accumulated onto the white dwarf via accretion and hydrogen and helium burning in shell sources in the dwarf's envelope. As soon as the WD mass exceeds the Chandrasekhar limit, a thermonuclear explosion occurs (or an explosion of the helium shell causes detonation of carbon in a dwarf with a mass below the Chandrasekhar limit), observable as a SN Ia [4]. The second model requires a close binary consisting of two CO dwarfs. Under the influence of gravitational-wave radiation, the binary's semi-major axis decreases, the dwarfs merge, and the resulting thermonuclear explosion is manifest as a SN Ia [5, 6] $]^{2}$. We consider the second scenario to be the main one [7].

Yungel'son [7] aims to prove that mergers are the principal, and possibly the only, source of SNe Ia. The accretion scenario is probably unable to explain SNe Ia: CO WDs are probably not able to accumulate enough gas to make their masses exceed the Chandrasekhar limit [7]. Observations also present evidence in favor of the WD-merger scenario [8, 9]. However, the viewpoint of [8, 9] are not shared by all authors [10]. Nevertheless, the main mechanism for SN Ia explosions in elliptical galaxies with ages in excess of approximately 200 million years is CO WD mergers [9]-[12]. The energies of some SN Ia correspond to masses of burned carbon and oxygen $\approx 2 M_{\odot}[13$, providing direct evidence in favor of the dwarf-merger model and confirming our estimates [1].

The merger rate of close binary WDs and its dependence on the star-formation history were studied in [11, 12]; the results of later studies are not very different (see, for example, [14, 15]). This may be because the WD merger rate is virtually independent of the merger mechanism if the merger time scale depends only on the initial semi-major-axis distribution for binary WDs [10]. Merger candidates are actively being searched for, and merger rates and the delay of the merger of degenerate close-binary components estimated (see, for instance, [16, 17]).

The role of the possible evolution of the energy of SNe Ia was studied ten years ago in [18], where several possible hypothetical luminosity evolutions for SNe Ia were considered. According to those computations, introducing even a small amount of evolution makes it very difficult to determine cosmological parameters such as the dark-energy density. We demonstrated in [1] that SNe Ia can be considered standard candles only after approximately one billion years of galaxy evolution ${ }^{3}$. In the course of their evolution, the mean energy of SNe Ia should decrease by approximately 10\%, and the difference between the highest and lowest energies of SNe Ia should be at least a factor of $1.5^{4}$ at both high and low redshifts. These obvious observational selection effects must be taken into account

\footnotetext{
${ }^{2}$ In this case, the combined mass of the dwarfs probably exceeds the Chandrasekhar limit.

${ }^{3}$ This result is independent of the star-formation history for the three versions of the star-forming function considered in [1, all stars are born simultaneously (star formation in an idealized elliptical galaxy), a constant star-formation rate (star formation in an idealized spiral galaxy), and the star formation history of 19 .

${ }^{4}$ It is a factor of two in the extreme case: a merger of two WDs with a combined mass approximately at the Chandrasekhar limit, or a merger of two WDs, each having a mass close to the Chandrasekhar limit. However, mergers of CO WDs with combined masses in excess of $2.1 M_{\odot}$ are rare. In addition, if we take into account the hypothetical possibility of explosions from CO WD mergers with combined masses below the Chandrasekhar limit, the difference between the highest and lowest energies of SNe Ia could be even greater than a factor of two. Studies of possible sub-Chandrasekhar scenarios for the origin of SNe Ia have now begun (see, for instance, [20, 21]).
} 
when estimating the parameters of the Universe's expansion using SNe Ia. The maximum brightness of such supernovae is not a universal constant, and has some dispersion. While we see "all" the closest SNe Ia, only the brightest are detected at increasing distances. This could imitate a brightening of SNe Ia with distance. The magnitude of this effect is comparable to the intrinsic brightness dispersion for this type of supernovae.

The brightness of a SNe Ia can be attenuated by absorption in matter around the supernova precursor, as well as absorption by dust in the supernovas parent galaxy; the principal characteristics of dust in the host galaxies of supernovae, total dust mass, distribution of dust in the galaxy, and dust composition can all vary in time. Redshiftdependent absorption by intragalactic gray dust could introduce errors into the extent to which SNe Ia can be considered standard candles; not taking into account absorption by gray dust makes photometric distances too large compared to a dust-free Universe and could give rise to an apparent "acceleration" of the expansion of the Universe [22]. According to [23], taking into account absorption by gray dust can make a SNe Ia fainter by $0 \cdot^{m} 08$ at a redshift of $z=1.7$, potentially capable of causing disagreement between Hubble diagrams calculated assuming a dark-energy density $\Omega_{\lambda} \simeq 0.7$ and observations of SNe Ia.

Absorption by intergalactic gray dust was suggested in [24, 25] as an explanation of the fainter magnitudes of supernovae at large distances, as an alternative to accelerating expansion of the Universe, almost immediately after the classic papers [2, 3] on observations of distant supernovae. In addition, a model with intergalactic dust was considered in [26], where it was suggested that intragalactic gas was not gray, and so could be accounted for by means of precision spectroscopy. However, later studies gave somewhat different results [27].

A comparison of the brightnesses of some 85 thousand quasars and the positions of about 20 million galaxies (based on the SDSS survey) demonstrated the presence of dust in intergalactic space, comparable in amount and composition to the dust in galactic disks [28. Problems related to the correction of supernova parameters taking into account absorption by this dust are discussed in [29], where the influence of gray dust is not considered. The authors of [28, 29] note that if the detected dust does not contain gray dust, they are able to provide an upper limit for absorption, but if gray dust is present, their computations give a lower limit for the influence of dust.

The example of the Milky Way shows that absorption in the parent galaxy can reach $1^{m}$, even in the polar direction. It also makes supernovae in disk galaxies fainter. For example, observations of stars in the Sloan Digital Sky Survey (SDSS) led to the discover and investigation of three absorbing clouds in the Galaxy, each hundreds of parsecs in size. The absorption in these clouds is $0^{m} .2-0^{m} .4$, and the clouds exhibit gray, almost wavelength-independent absorption in the spectral range of the survey [27]. When estimating the evolution of the brightness of SNe Ia in time, we must remember that the gas component of the galaxies containing these supernovae also evolves. This introduces additional uncertainty into brightness estimates for supernovae. The higher infrared luminosities of distant $(z \approx 1)$ galaxies indicate the presence of high dust contents in young galaxies (e. g., [30]).

We can put the following limit on radiation absorption by dust in an elliptical galaxy with a mass comparable to that of the Galaxy. Let the total mass lost by red supergiants 
be $\sim 1 M_{\odot}$ per year. The mass loss time scale for an elliptical galaxy is $\sim 10^{8}$ years. Thus, the stationary amount of gas in the galaxy is $\sim 10^{8} M_{\odot}$. In this case, the absorption for the usual abundance of dust will be $\approx 0^{m} .1$, if we assume the same efficiency for dust absorption in elliptical and disk galaxies.

To estimate the absorption of light from supernovae in disk galaxies, we applied the evolutionary model of [31]-[33]. The assumed mass and radius of the model galaxy were $2 \cdot 10^{11} M_{\odot}$ and $\sim 10^{4} \mathrm{pc}$. We estimate the star-formation rate from the condition that the gaseous disk be fully ionized by young massive stars [31]. The thickness of the gas disk is estimated from the condition that the rate at which energy is supplied to the turbulent motion of the galaxys gas by supernovae (II and Ib,c) be the same as the dissipation rate of the turbulent motion of this gas [31]. In this case, the rate of supernova outbursts is determined by the star-formation rate in the model galaxy, and the dissipation of the turbulent motion of the interstellar gas is determined by collisions between interstellar gas clouds.

Modeling demonstrated that the star-formation rate in a disk galaxy varies considerably in time [32. The star-formation rate increases during the several first billion years. In a galaxy similar to ours, the star-formation rate reaches a maximum of $\sim 10^{2} M_{\odot}$ per year at an age of $2-4$ billion years. During the subsequent evolution, the star-formation rate gradually decreases to its current level. The model takes into account gas enrichment with heavy elements, making it possible to follow the time evolution of the gas components optical depth when checking the mass of the gaseous component. Knowing this, we are able to estimate the time evolution of the apparent brightnesses of supernovae, assuming that all are located in the planes of their host galaxies, which are orthogonal to the line of sight. In this way, we can take into account the effect of absorption of light from supernovae in disk galaxies. For the current epoch, this amounts to $\approx 0^{m} .5$, and increases for young galaxies with high gas and dust contents. Dust is thus able to attenuate the brightness of SNe Ia.

It is especially important to note that we are concerned here with gray dust, whose absorption is wavelength-independent. The existence of such dust in the galactic plane is now beyond doubt [27]. Fine dust causes reddening of light and thus, in principle, can be taken into account. It should also be borne in mind that modeling of the time evolution of a galaxy's optical depth remains uncertain, and the optical depth itself cannot be estimated better than to within a factor of two [31, 32]. Gray-dust light absorption occurs independently of the supernova model that is preferred. We will assume here that the amount of gray dust in a parent galaxy of a SN Ia is proportional to its total amount of dust.

\section{MODEL EVOLUTION OF SNe Ia BRIGHTNESS WITH INCREASING REDSHIFT}

In this study, we analyze the computational results used to plot Figs. 1-3 in $[1]^{5}$. We used these computations to derive the mass distributions of merging WDs [1] (Fig. 1) and

\footnotetext{
${ }^{5}$ The computations of [1] were based on the "Scenario Machine". A description of the code can be found in [34, 35], and of the population synthesis, in [36].
} 
the dependence of the mean mass of merging WDs on the time elapsed since the onset of star formation [1] ( Fig. 2). Virtually independent of the form of the star-formation functions, the mean mass of merging $\mathrm{CO}$ WDs with combined masses in excess of the Chandrasekhar limit decreases from $\approx 1.9 M_{\odot}$ to $\approx 1.7 M_{\odot}$ within about one billion years after the onset of star formation, after which the mean mass of merging WDs remains practically the same.

The aim of this study is to provide a theoretical analog of a Hubble diagram [2] (Figs. $4,5)$ taking into account the brightness evolution of SN Ia and the evolution of dust absorption, using the theoretical model presented in [33]. We assume that the absolute magnitude of a SN Ia depends on the mean mass of the merging CO WDs as

$$
M=C-2.5 \cdot \log M_{\Sigma}
$$

where $M$ is the absolute magnitude of the SN Ia, $M_{\Sigma}$ the combined mass of the merging CO WDs, and $C$ is a constant. Thus, the higher the combined mass of the merging dwarfs, the brighter the supernova. Using 1 to re-calculate the data plotted in Fig. 2 of [1], we obtained the dependence of the absolute magnitude of a SN Ia on the time since the onset of the formation of the corresponding stellar population.

The resulting dependence of the absolute magnitude on the time from the onset of the galaxy's evolution until the supernova outburst must be transformed into the dependence of the observed SNe Ia magnitudes on the observed redshifts for the host galaxies. These computations used Eq. 20 (to calculate the time-redshift relation) and 28 (to calculate the photometric distance-redshift relation) from [37]. The free parameters in these formulas are the density of dark energy $\Omega_{\lambda}$, the matter density $\Omega_{M}$, the total density of the Universe $\Omega_{t o t}$, and the current Hubble constant $H_{0}$. Another free parameter of our problem is the redshift $z^{*}$ corresponding to the onset of star formation in galaxies.

The last stage of our computations is to calculate the dependence of $\Delta(m-M)$ on redshift $z$ :

$$
\Delta(m-M)=\left(m_{1}-C\right)-\left(m_{2}-C\right)=m_{1}-m_{2},
$$

where $m$ and $M$ are the apparent and absolute magnitudes, $m_{1}$ the apparent magnitude calculated taking into account the time evolution of the mean mass of the merging WDs, and $m_{2}$ the apparent magnitude calculalated assuming that a SN Ia is a standard candle with absolute magnitude $C$.

We took the dependence for light absorption by gray dust from [33] (Fig. 2 presents the dependence of $A_{v}$ on the redshift $z$; we used the curve corresponding to a disk galaxy with the mass-radius relation $M \sim R^{2}$, see [33] for details). The redshift dependence of the absorption in this figure was derived using the formula $t(z)=\frac{2}{3 H_{0}(1+z)^{3 / 2}}$ relating the time $t(z)$ elapsed since the Big Bang and the redshift $z$. We place this relation on the Hubble diagram in order to qualitatively illustrate the evolution of dust absorption in disk galaxies, compared to the accelerated fading of supernovae at redshifts $0.5-1$. Note also that absorption by dust grains of any size was considered in [33]; that paper contains no estimate of the fraction of gray absorption due to grains with sizes in excess of $1 \mu \mathrm{m}$. 


\section{RESULTS}

The results of our computations are presented in Figs. 1 and 2, The values computed using 2 are plotted versus redshift. The magnitude $m_{2}$ used in 2 to calculate the curves in Figs. 1 and 2 is the dependence of the apparent magnitude $m$ on the redshift $z$, assuming that all SN Ia are standard candles with absolute magnitude $C$ and that they do not undergo any absorption (at least no time-variable absorption). This relation corresponds to the horizontal straight line at zero magnitude (curve 3 ). The magnitude $m_{1}$ is the dependence of $m$ on $z$ taking into account one of the studied effects.

Curve 1 describes the dependence of $\Delta(m-M)$ on $z$, assuming that either absorption plays no role or it is constant in time, and that the absolute magnitude evolves in time from its maximum value at the beginning of the evolution to its minimum, approximately constant, value after about a billion years of stellar evolution in galaxies. The adopted cosmological parameters are $\Omega_{\lambda}=0, \Omega_{M}=0.2, \Omega_{t o t}=0.2, H_{0}=70 \mathrm{~km} \mathrm{~s}^{-1} \mathrm{mpc}^{-1}$, $z^{*}=10$.

Curve 2 describes the dependence of $\Delta(m-M)$ on $z$, assuming that evolutionary effects related to the mass of the merging dwarfs and light absorption in galaxies play no role; here, we adopted $\Omega_{\lambda}=0.7, \Omega_{M}=0.3, \Omega_{t o t}=1, H_{0}=70 \mathrm{~km} \mathrm{~s}^{-1} \mathrm{mpc}^{-1}$.

The line 3 corresponds to a model without evolutionary effects and with no influence from dark energy $\left(\Omega_{\lambda}=0, \Omega_{M}=0.2, \Omega_{t o t}=0.2, H_{0}=70 \mathrm{~km} \mathrm{~s}^{-1} \mathrm{mpc}^{-1}\right)$.

Curve 4 describes the evolution of light absorption by dust. This relation was taken from [33] (Fig. 2 displaying the dependence of the absorption $A_{v}$ on the redshift $z$, adopting the curve corresponding to a disk galaxy with $M \sim R^{2}$ ).

Figure 1 shows that dust absorption is able to attenuate the brightness of SN Ia by as much as $\approx 1^{m}$.3. This absorption is reached at redshifts $z \approx 3$. The evolution of the mean mass of the merging CO WDs begins to be appreciable at $z>2$ and leads to an increased brightness of SNe Ia in the past compared to the current epoch. The mean apparent magnitude can decrease by $\simeq 0^{m} .2$ at $z \gtrsim 8$, in full agreement with the possibility that the mean mass of merging CO WDs (and, according to our assumptions, the mean energy of SNe Ia) was higher at the beginning of galaxy evolution, in the distant past, compared to the current epoch.

To make it easier to compare our results to Figs. 4 and 5 from [2], Fig. 2 isolates the redshift range between 0.01 and 1 . The evolution of the mean mass of the merging dwarfs essentially cannot influence the results in this redshift range. Dust absorption attenuates the brightness of supernovae much more strongly than if we allow for a possible contribution from dark energy (Figs. 4 and 5 in [2]), but recall that [33] presents data for absorption by any dust, including dust whose influence can be taken into account based on reddening. In order to explain the accelerated fading of SNe Ia at $z=0.5-1$ as due to evolution of gray-dust absorption in disk galaxies, it is sufficient to suppose the amount of gray dust is proportional to the total amount of gas in the host galaxies of supernovae, and comprises several tens of percent of the total dust absorption in these same galaxies. 


\section{CONCLUSIONS}

We have considered two possible channels for the "standard" behavior of the SNe Ia brightness being violated. The first is the time evolution of the mean mass of merging $\mathrm{CO}$ WDs, which are the precursors of SNe Ia. The second is the time evolution of absorption by gray dust in the SNe Ia host galaxies.

According to our computations, the mean energy of SNe Ia should increase from $z>2$ and become significantly higher at $z \gtrsim 8$ due to the fact that, on average, mergers involved higher-mass WDs during the early evolutionary stages of the Universe than at the current epoch. Such distant supernovae have not yet been discovered. Thus, the conclusion that the expansion of the Universe is accelerating based on observations of SNe Ia at redshifts to $z \simeq 1$ made in [2, 3] will not be affected by evolution of the mean mass of the merging dwarfs. At the same time, it should always be borne in mind during such estimation that our models, which are based on population syntheses, assume that the objects whose evolution we are studying (galaxies) were born at some time in the past (in the current study, at $z^{*}=10$ ), then evolved. However, if a burst of star formation occurred at some epoch after $z^{*}$, conclusions about the increase of the mean SNe Ia energy should be related to the time since that burst. Independent of the population's age, there will exist some dispersion in supernova parameters, because the combined mass of the merging dwarfs can vary within a factor of approximately 1.5 .

According to the model considered here, the evolution of radiation absorption by galactic dust can give rise to a much stronger attenuation of the brightness of distant supernovae than the assumption that dark energy is present in the Universe. At the same time, the absorption described by curve 4 in Figs. 1 and 2 is the sum of the absorption that can, in principle, be taken into account based on reddening and gray absorption, which cannot. By varying the model using its intrinsic parameters and introducing the required fraction of gray dust, we are able, in principle, to achieve an essentially ideal agreement between curves 2 and 4 over a wide redshift range (this reasoning is very similar to the considerations presented in [24]-[26]). However, this approach is often criticized: fine tuning of the model parameters is believed to be able to reproduce any results, and interpretation of the phenomenon in this way seems unattractive [38].

During the analysis of the SDSS-II supernova survey (and of the light curves of these supernovae), SNe Ia were found to be approximately $0^{m} .1$ brighter in quiet galaxies than in galaxies with active star formation $[39]^{6}$. The characteristics of absorption by dust are also different in galaxies with and without active star formation [39]. A dependence of the SNe Ia luminosity on galaxy type was also noted in [40].

We suggest the following interpretation of our results. Figure 2 shows that absorption by dust in disk galaxies grows at essentially the same rate as the rate of dimming of supernovae due to accelerated expansion of the Universe. Hence, we suggest that the decrease in the observed brightness of supernova (on average, and this is important) is due primarily to radiation absorption by gray dust, whose influence cannot at this time be taken into account using standard procedures for correcting for absorption. However, further increase of the redshift results in increasing absorption, causing still stronger brightness attenuation of supernovae compared to a model without dust. At the same

\footnotetext{
${ }^{6}$ Based on analysis of supernovae with redshifts up to $z \simeq 0.2-$ see [39, Fig. 3 .
} 
time, some distant supernovae are found on the curve corresponding to decelerating, rather than accelerating, expansion of the Universe (see, for instance, Fig. 10 in [41]). This could be considered unequivocal evidence against the idea that the observed supernova fading can be explained by time-evolving absorption by gray dust ${ }^{7}$ if all host galaxies of SNe Ia were disk galaxies with active star formation. However, this is not the case: for example, the parent galaxy of SN 1997ff (one of the most distant supernovae, used as a standard) is elliptical [42].

As noted above, compared to disk galaxies, absorption in elliptical galaxies is insignificant, and apparently does not evolve over a considerable time interval, comparable to the Hubble time. Note also that population syntheses of the evolution of close binaries (e.g., Fig. 1 in [12] or Fig. 2 in [15]) show that the rate of SNe Ia outbursts in an elliptical galaxy decreases by approximately a factor of two between 1 and 10 billion years after the formation of the elliptical galaxy. This means that the probability of observing a SNe Ia in an elliptical galaxy, rather than a disk galaxy, was much higher in the past (among supernovae with the highest $z$ ) than at the current epoch (for low $z$ ). This can explain the observed lack of "accelerated" supernovae for redshifts $z>1$.

Thus, when using SNe Ia as cosmological standard candles, it is necessary to take into account not only model characteristics of the supernovae, but also evolution of absorption by dust, including gray dust, in the supernova host galaxies. It is preferable to use supernovae in galaxies without star formation as distance indicators ${ }^{8}$. Supernova outbursts in galaxies with active current star formation can serve as probes for studies of the evolution of absorption in such galaxies (including gray absorption ${ }^{9}$ ).

Variations in the combined masses of binary degenerate dwarfs with ages comparable to the age of the Universe do not significantly change the brightness of SNe Ia explosions. It follows from the discussions above that analysis of the evolution of SNe Ia brightnesses aimed at improving cosmological models must be carried out separately for elliptical and spiral galaxies. SNe Ia in elliptical galaxies seem to provide a more reliable cosmological tool than SNe Ia in spiral galaxies, since the latter contain dust. Searches for and studies of very distant SNe Ia, at redshifts $z>2$ are especially important.

\section{ACKNOWLEDGMENTS}

This work was supported by a grant from the President of the Russian Federation for the State Support of Young Russian PhDs (MK-142.2009.2), the Departmental Targeted Analytical Program The Development of the Scientific Potential of Higher Education (RNP-2.1.1/2906), the State Program of Support for Leading Scientific Schools of the Russian Federation (NSh-7179.2010.2), the Basic Research Program of the Department of Physical Studies of the Russian Academy of Sciences Active Processes and Stochastic Structures in theUniverse, the Basic Research Program of the Presidium of the Russian Academy of Sciences Origin, Structure, and Evolution of Objects in the Universe, the

\footnotetext{
${ }^{7}$ Or, in principle, by any dust that was not duly taken into account.

${ }^{8}$ Note that some authors (e.g., 43]) believe that available observations, including those for SNe Ia, are consistent with a wide range of models of the Universe.

${ }^{9}$ Recall that gray absorption has been discovered in the Milky Way, and can reach several tenths of a magnitude [27.
} 
Russian Foundation for BasicResearch (project 10-02-00231), and an Integration project of the Siberian Division of the Russian Academy of Sciences (No. 103).

\section{References}

1. A. I. Bogomazov and A. V. Tutukov, Astron. Zh. 86, 240 (2009) [Astron. Rep. 53, $214(2009)]$.

2. A. G. Riess, A. V. Filippenko, P. Challis, et al., Astron. J. 116, 1009 (1998).

3. S. Perlmutter, G. Aldering, G. Goldhaber, et al., Astrophys. J. 517, 565 (1999).

4. J. Whelan, I. Iben, Astrophysical Journal, v. 186, pp. 1007-1014 (1973).

5. I. Iben, A. Tutukov, v. 54, p. 335-372 (1984).

6. A. V. Tutukov and L. R. Yungelson, Astron. Zh. 57, 1266 (1980) [Sov. Astron. 24, $729(1980)]$.

7. L. R. Yungelson, Pis'ma Astron. Zh. 36, 823 (2010) [Astron. Lett. 36, 780 (2010)].

8. R. Di Stefano, Astrophysical Journal, v. 712, pp. 728-733 (2010).

9. M. Gilfanov, A. Bogdan, Nature, v. 463, pp. 924-925 (2010).

10. V. M. Lipunov, I. E. Panchenko, and M. V. Pruzhinskaya, New Astronomy, 16, 250 (2011).

11. A. V. Tutukov, L. R. Yungelson, Monthly Notices of the Royal Astronomical Society, v. 268, pp. 871-879 (1994).

12. H. E. Jorgensen, V. M. Lipunov, I. E. Panchenko, K. A. Postnov, M. E. Prokhorov, Astrophysical Journal, v. 486, p. 110-116 (1997).

13. J. M. Silverman, M. Ganeshalingam, W. Li, et al., Mon. Not. R. Astron. Soc. [Monthly Not. Roy. Astron. Soc. 410, 585 (2011)].

14. A. J. Ruiter, K. Belczynski, C. Fryer, Astrophysical Journal, v. 699, pp. 2026-2036 (2009).

15. N. Mennekens, D. Vanbeveren, J. P. De Greve, E. De Donder, Astronomy and Astrophysics, v. 515, id. A89 (2010).

16. M. Kilic, W. R. Brown, C. Allende Prieto et al., Astrophysical Journal, v. 716, pp. 122-130 (2010).

17. D. Maoz, K. Sharon, and A. Gal-Yam, Astrophys. J. 722, 1879 (2010).

18. P. S. Drell, T. J. Loredo, I. Wasserman, Astrophysical Journal, v. 530, pp. 593-617 (2000). 
19. E. P. Kurbatov, A. V. Tutukov, and B. M. Shustov, Astron. Zh. 82, 573 (2005) [Astron. Rep. 49, 510 (2005)].

20. S. Sim, F. Ropke, W. Hillebrandt, et al., Astrophys. J. Letters, v. 714, pp. L52-L57 (2010).

21. A. Ruiter, K. Belczynski, S. Sim, et al., eprint arXiv:1009.3661 (2010).

22. B. W. Holwerda, Mon. Not. of the Roy. Astron. Soc., v. 386, pp. 475-480 (2008).

23. P. Corasaniti, Mon. Not. Roy. Astron. Soc., v. 372, pp. 191 (2006).

24. A. Aguirre, The Astrophysical Journal, v. 512, pp. L19-L22 (1999).

25. A. Aguirre, The Astrophysical Journal, v. 525, pp. 583-593 (1999).

26. A. Goobar, L. Bergstrom, E. Mortsell, Astronomy and Astrophysics, v. 384, pp. 1-10 (2002).

27. E. Gorbikov, N. Brosch, Monthly Notices of the Royal Astronomical Society, v. 401, pp. 231-241 (2010).

28. B. Menard, R. Scranton, M. Fukugita, Monthly Notices of the Royal Astronomical Society, v. 405, pp. 1025-1039 (2010).

29. B. Menard, M. Kilbinger, R. Scranton, Monthly Notices of the Royal Astronomical Society, v. 406, pp. 1815-1820 (2010).

30. V. Buat, E. Giovannoli, D. Burgarella et al., Monthly Notices of the Royal Astronomical Society, v., p. (2010).

31. C. Firmani, A. Tutukov, Astron. Astrophys., v. 264, p. 37 (1992).

32. D. Z. Wiebe, A. V. Tutukov, and B. M. Shustov, Astron. Zh. 75, 3 (1998) [Astron. Rep. 42, 1 (1998)].

33. A. A. Kabanov, A. V. Tutukov, and B. M. Shustov, Astron. Zh. 88, 218 (2011) [Astronomy Reports 55, 193 (2011)].

34. V. M. Lipunov, K. A. Postnov, M. E. Prokhorov, The Scenario Machine: Binary Star Population Synthesis, Astrophysics and Space Physics Reviews, vol. 9, Harwood academic publishers, ed. R. A. Sunyaev (1996).

35. V. M. Lipunov, K. A. Postnov, M. E. Prokhorov, and A. I. Bogomazov, Astron. Zh. 86, 985 (2009) [Astron. Rep. 53, 915 (2009)].

36. S. B. Popov and M. E. Prokhorov, Usp. Fiz. Nauk 177, 1179 (2007) [Phys. Usp. 50, 1123 (2007)].

37. V. Sahni, A. Starobinsky, International Journal of Modern Physics D, v. 9, pp. 373-443 (2000). 
38. A. G. Riess, L.-G. Strogler, S. Casertano, et al., Astrophys. J., v. 659, p. 98-121 (2007).

39. H. Lampeitl, M. Smith, R. Nichol, et al., Astrophys. J., v. 722, pp. 566-576 (2010).

40. M. Sullivan, A. Conley, D. A. Howell, et al., Mon. Not. Roy. Astron. Soc., v. 406, pp. 782-802 (2010).

41. M. Kowalski, D. Rubin, G. Aldering, et al., Astrophys. J., v. 686, pp. 749-778 (2008).

42. A. G. Riess, P. Nugent, R. Gilliland, et al., Astrophys. J., v. 560, 49-71 (2001).

43. R. Vishwakarma, Mon. Not. Roy. Astron. Soc., v. 345, pp. 545-551 (2003). 


\section{FIGURE CAPTIONS}

Fig. 1: Difference between the magnitude-redshift dependences taking into account the effects studied here (time evolution of the absorption and the mean mass of merging WDs) and assuming standard SNe Ia that are not subject to variable absorption in time, $\Delta(m-M)$. Curve 1 assumes that absorption does not play any role and that the absolute magnitude evolves in time from a maximum value at the beginning of the evolution to a minimum, approximately constant, value after about one billion years of stellar evolution in galaxies (for $\Omega_{\lambda}=0, \Omega_{M}=0.2, \Omega_{t o t}=0.2, H_{0}=70 \mathrm{~km} \mathrm{~s}^{-1} \mathrm{Mpc}^{-1}, z^{*}=10$ ). Curve 2 assumes that absorption does not play any role and the absolute magnitudes experience no evolutionary effect $\left(\Omega_{\lambda}=0.7, \Omega_{M}=0.3, \Omega_{t o t}=1, H_{0}=70 \mathrm{~km} \mathrm{~s}^{-1} \mathrm{Mpc}^{-1}\right)$. The line 3 corresponds to a model without evolutionary effects $\left(\Omega_{\lambda}=0, \Omega_{M}=0.2, \Omega_{\text {tot }}=0.2\right.$, $\left.H_{0}=70 \mathrm{~km} \mathrm{~s}^{-1} \mathrm{Mpc}^{-1}\right)$. Curve 4 represents the evolution of light absorption by dust, taken from 33 . (Fig. 2 displaying the dependence of $A_{v}$ on the redshift $z$; the curve corresponding to a disk galaxy with $M \sim R^{2}$ ).

Fig. 2: Same as Fig. 1 for redshifts between 0.01 and 1. 


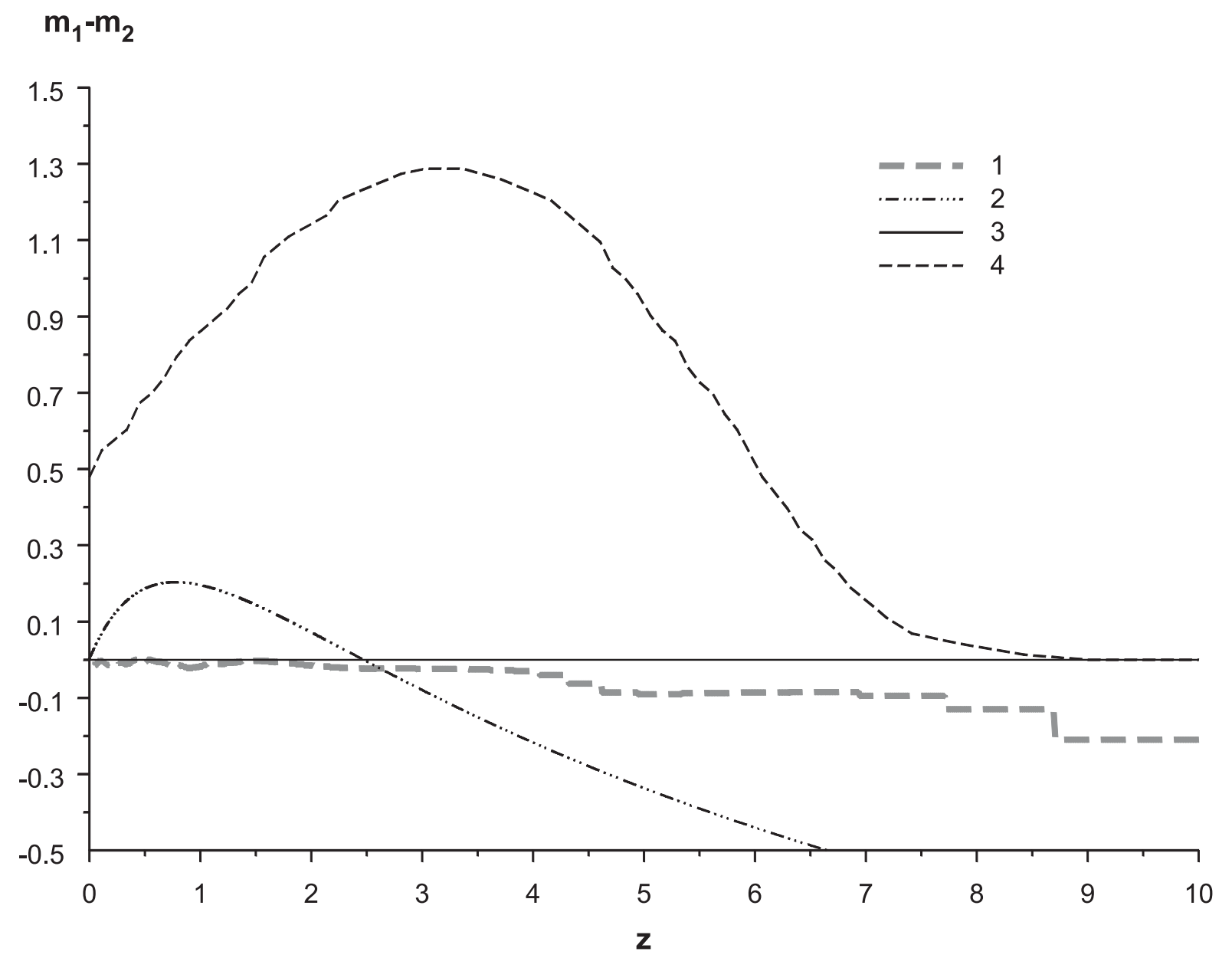

Figure 1: 


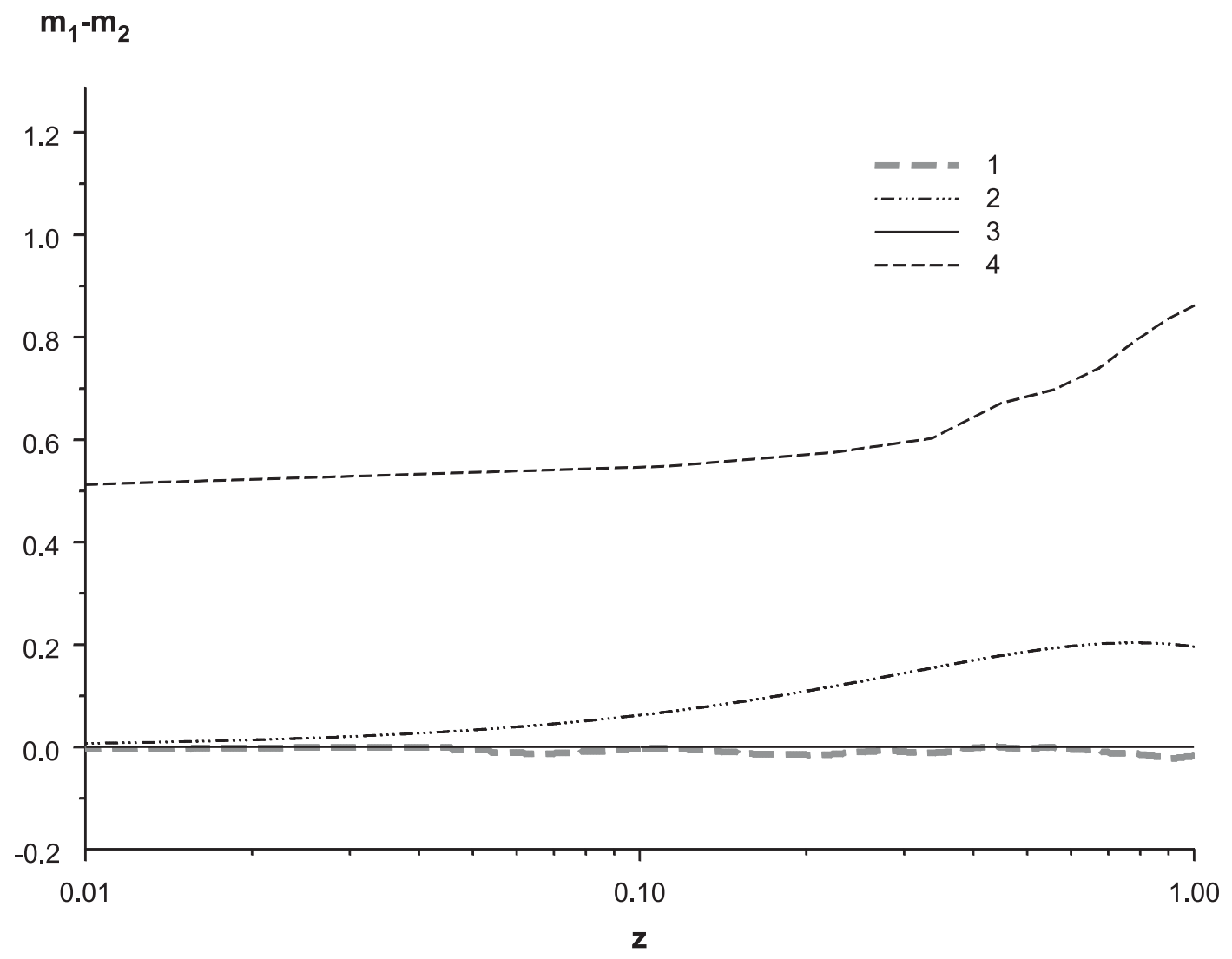

Figure 2: 